\title{
Utility of a Ruler for Reconstructed Auricle Positioning
}

\author{
Pengfei Sun (iD \\ Meng Lu \\ Changchen Wang \\ Bo Pan (i)
}

Department of Plastic Surgery, Plastic Surgery Hospital, Chinese Academy of Medical Sciences and Peking Union Medical College, Beijing, I00I44, People's Republic of China
Correspondence: Bo Pan Department of Plastic Surgery, Plastic Surgery Hospital, Chinese Academy of Medical Sciences and Peking Union

Medical College, No. 33 Badachu Road, Shijingshan District, Beijing, 100144,

People's Republic of China

Tel +86138108559/2

Email zbzbzhc@I63.com
Purpose: This study evaluated the utility of a ruler for reconstructed article positioning. Methods: Forty-seven patients with unilateral microtia were selected from August 2020 to September 2021.

Results: The linear distance from the highest point of the reconstructed auricle to the central axis of the nose was not significantly different from the distance from the highest point of the normal contralateral auricle to the central axis of the nose $(\mathrm{P}>0.05)$. The distance from the lowest point of the reconstructed auricle to the central axis of the nose was not significantly different from the distance from the lowest point of the normal auricle to the central axis of the nose $(\mathrm{P}>0.05)$. The linear distance from the highest to the lowest point of the reconstructed auricle was not significantly different from the distance from the highest to the lowest point of the normal auricle $(\mathrm{P}>0.05)$. These results indicate that the reconstructed auricle was symmetrical to the contralateral ear.

Conclusion: The positioning ruler evaluated in this study is simple, easy to use, accurate, and non-invasive.

Keywords: microtia, reconstructed auricle, ruler, ear

\section{Introduction}

Congenital microtia is a genetic disease with unknown etiology. ${ }^{1}$ Its main clinical symptom is auricular dysplasia, usually accompanied by external auditory canal atresia, and middle ear and maxillofacial deformities. ${ }^{2}$ The global prevalence of congenital microtia is 0.80 to 4.53 births per 10,000 births. $^{3}$ Despite the low prevalence, this entity strongly affects children's facial appearance and mental health. ${ }^{4}$ The most effective method for treating congenital microtia is auricular reconstruction. After surgical intervention, the reconstructed and contralateral ear should have the same size and position. In 1994, Nagata performed quantitative measurements to determine the best position for the reconstructed auricle. ${ }^{5}$ In 1997 , Nagata and Akira Yamada invented the ear-positioning template to locate the reconstructed auricular. ${ }^{6}$ Nonetheless, these methods they used are prone to measurement errors.

Chen et $\mathrm{al}^{7}$ used multi-slice spiral computed tomography (CT) to collect patients' skull data and used a 3D printer to make a 3D template for positioning the reconstructed auricle. Kohei Umekawa ${ }^{8}$ used 3D CT to determine the best site for auricular reconstruction. Walsh et $\mathrm{al}^{9}$ positioned the reconstructed auricle using silicone molds. These methods are technically demanding, difficult to operate, and are not conducive to the popularization and application of these technologies. In the treatment of 
congenital microtia, our otoplasty team developed a ruler to determine the position of the reconstructed auricle.

\section{Patients and Methods}

\section{Clinical Data}

Forty-seven patients ( 29 men and 18 women) with unilateral microtia ( 20 on the left side and 27 on the right) were selected from August 2020 to September 2021, and the position of the reconstructed auricle was assessed in the Plastic Surgery Hospital using a ruler. The mean age of the patients was $10.77 \pm 2.64$ years, and the mean follow-up period was 8.68 \pm 2.40 months. This study was approved by the Medical Ethics Committee of our hospital. All study patients and their families signed an informed consent form. All procedures involving human participants conformed to the ethical guidelines of the institutional and/or national research committee and the 1964 Declaration of Helsinki.

\section{Inclusion and Exclusion Criteria}

The inclusion criteria were patients with auricular deformities, patients in good health, and patients who agreed with the use of a ruler to determine the position of the reconstructed auricle.

The exclusion criteria were the presence of bilateral microtia, other head and face deformities, diseases that could affect surgical outcomes, severe hemifacial microsomia, serious heart, kidney, liver, and other organ diseases that could affect surgical outcomes, severe craniofacial asymmetry, and patients who did not agree with the use of a ruler to determine the position of the reconstructed auricle.

\section{The Structure of the Ruler is Shown in} Figure I

The ruler has a main body (Figure 1C) and two arms (Figure 1A and B). The main body is composed of retractable stainless-steel rods. An instrument that rotates over the stainless-steel rod is mounted on this section (Figure 1D). The two arms are stretchable stainless-steel rods. The section that connects the two arms with the main body are gears with scale (Figure 1E) that can be adjusted to the parallel position of the two arms.

\section{Use of the Ruler}

The patients were placed in the supine position during measurements, with the head straight and stationary, to avoid measurement errors. First, the upper edge of the reconstructed auricle (Figure 2) was located using the ruler based on the upper edge of the normal contralateral auricle (Figure 3). Then, the lower edge of the reconstructed auricle (Figure 4) was located based on the lower edge of the normal auricle (Figure 5). The line from the lowest to the highest point of the reconstructed auricle (Figure 6) was determined according to the line from the lowest to the highest point of the earlobe of the normal auricle (Figure 7). That is, the inclined angle of the reconstructed auricle. In this way, we determined the symmetric position between the reconstructed auricle and the contralateral ear by using the positioning ruler (Figure 8). During measurements, the main section of the ruler was kept at a horizontal position using a level (Figure 1D) to avoid measurements errors. In clinical

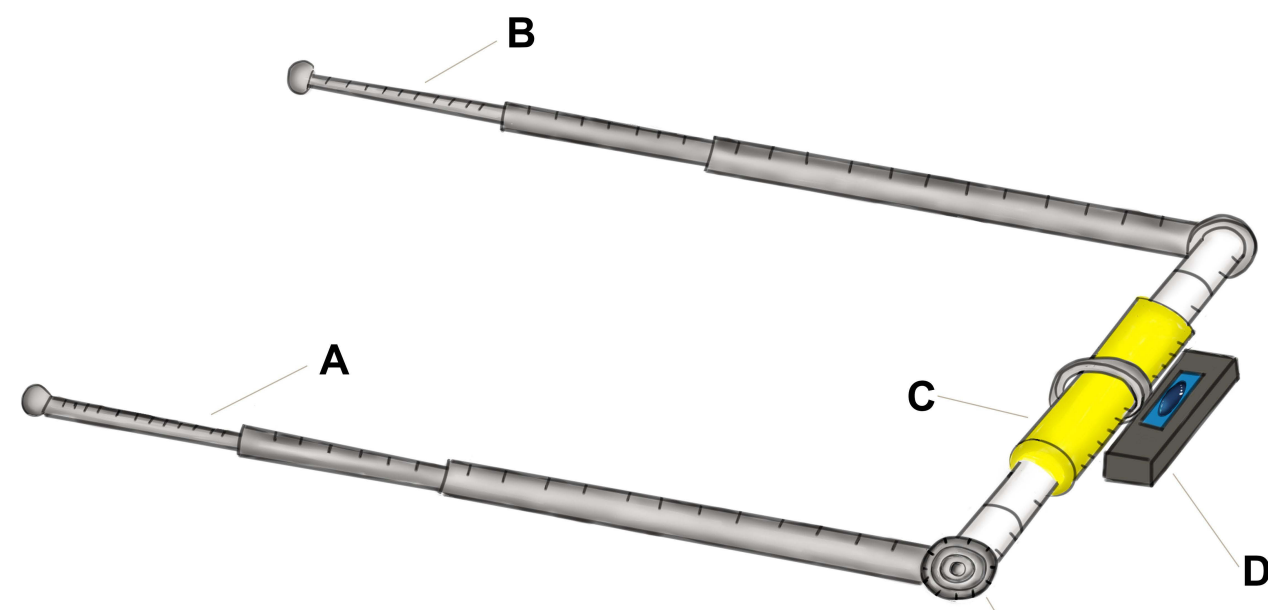

E

Figure I Structure of the ruler. A and B: arms; C: main body; D: level; E: the gear with scale. 


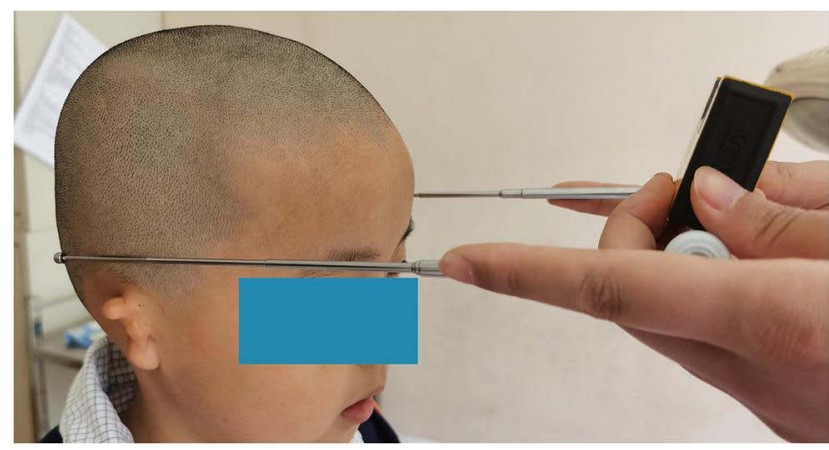

Figure 2 Upper edge of the reconstructed auricle.

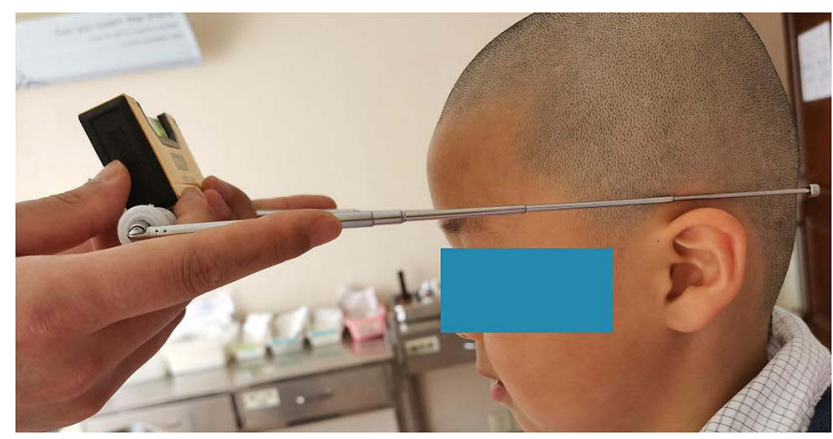

Figure 3 Upper edge of the normal contralateral auricle.

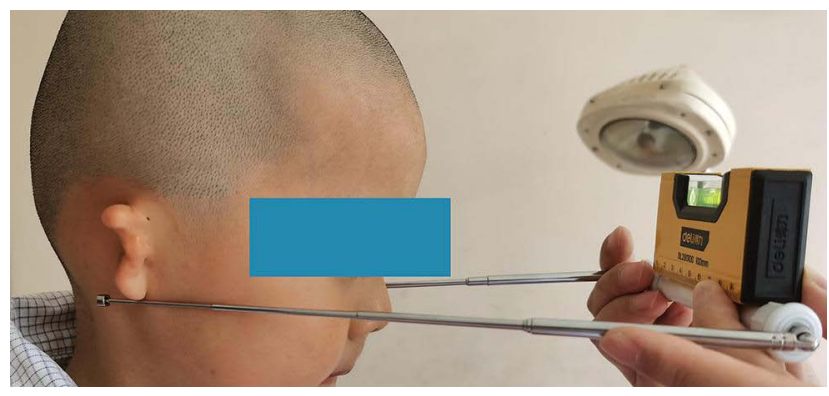

Figure 4 Lower edge of the reconstructed auricle.

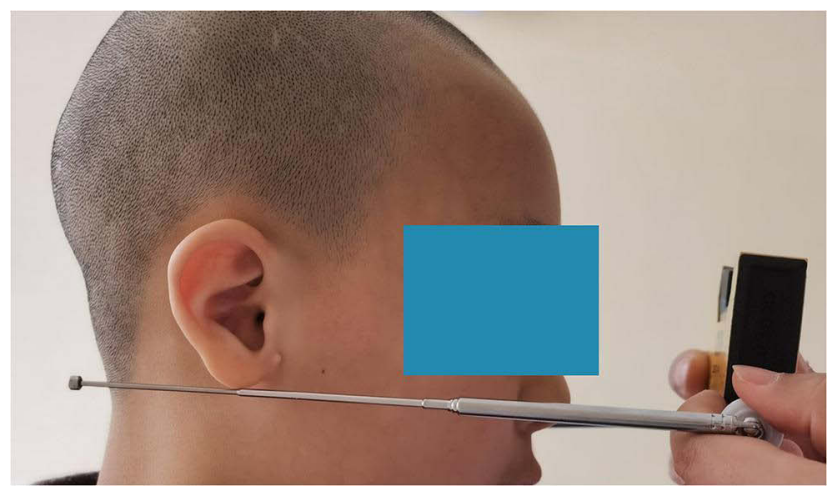

Figure 5 Lower edge of the normal contralateral auricle.

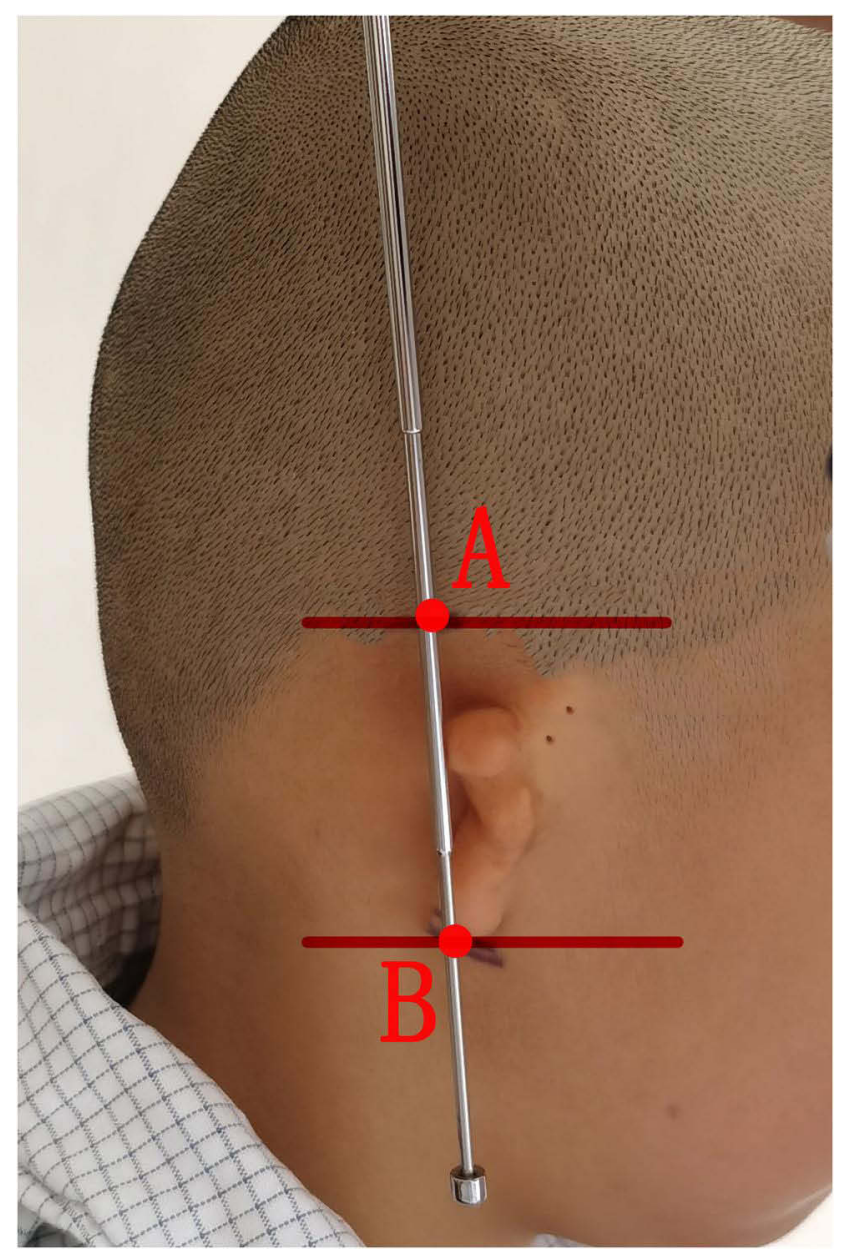

Figure 6 The line from the lowest to the highest point of the reconstructed auricle. (A) highest point of the reconstructed auricle; (B) lowest point of the reconstructed auricle.

application, the stability of patient's head is good because the measurement process takes a short time.

\section{Evaluation Criteria}

The distance from the highest point of the reconstructed auricle to the central axis of the nose was compared with the distance from the highest point of the normal auricle to the central axis of the nose. The distance from the lowest point of the reconstructed auricle to the central axis of the nose was compared with the distance from the lowest point of the normal auricle to the central axis of the nose. The distance from the highest to the lowest point of the reconstructed auricle was compared with the distance from the highest to the lowest point of the normal auricle. In order to ensure the accuracy of the data, patients 


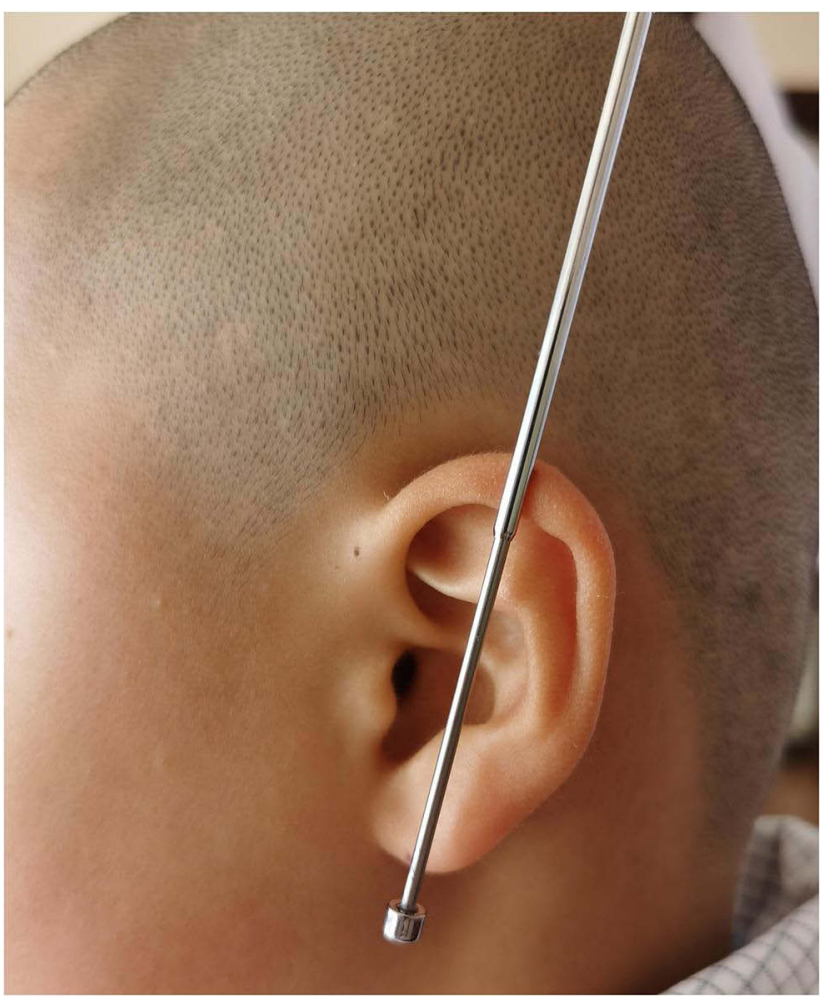

Figure 7 The line from the lowest to the highest point of the normal auricle. were placed in supine position during measurement to ensure the stability of the patients' heads (Figure 9).

\section{Statistical Analysis}

Data were analyzed using SPSSAU (https://spssau.com/). Differences in categorical variables were analyzed using the chi-squared test and expressed as numbers and percentages. Continuous variables are presented as means \pm SD. The normality of the distributions was assessed using the Shapiro-Wilk test. Normally and non-normally distributed data were analyzed using a $t$-test and Wilcoxon signedrank test, respectively. P-values of less than 0.05 were considered statistically significant.

\section{Results}

A total of 47 patients were included in the study. The average distance from the highest point of the reconstructed auricle to the central axis of the nose was $14.12 \pm 0.52 \mathrm{~cm}$. The average distance from the highest point of the normal auricle to the central axis of the nose was $14.08 \pm 0.45 \mathrm{~cm}$. The average distance from the lowest point of the reconstructed auricle to the central axis of the nose was $12.71 \pm 0.31 \mathrm{~cm}$. The average

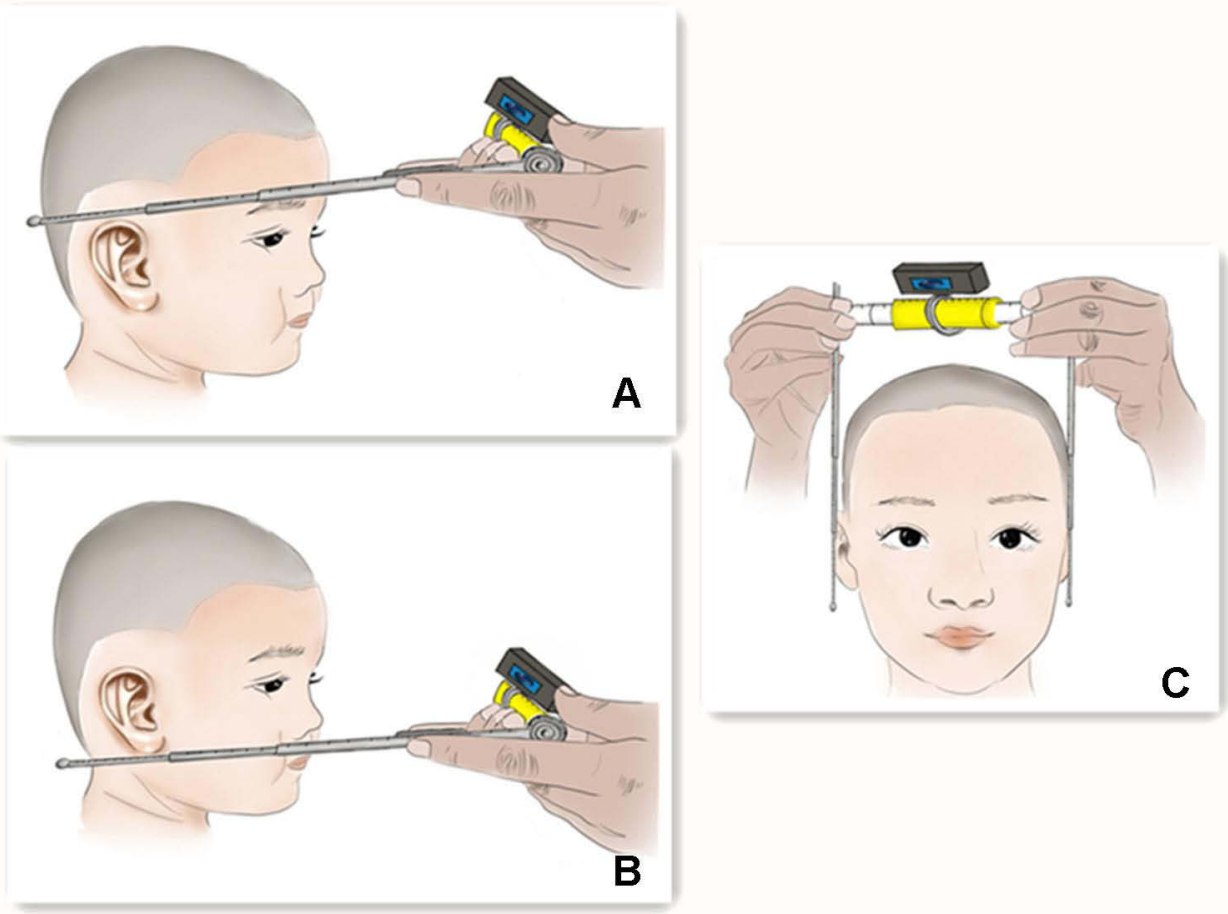

Figure $\mathbf{8}$ Use of the ruler. (A) the first step is to locate the highest point of the ear; (B) the second step is to locate the lowest point of the ear; (C) the third step is to determine the inclined angle of the ear. 


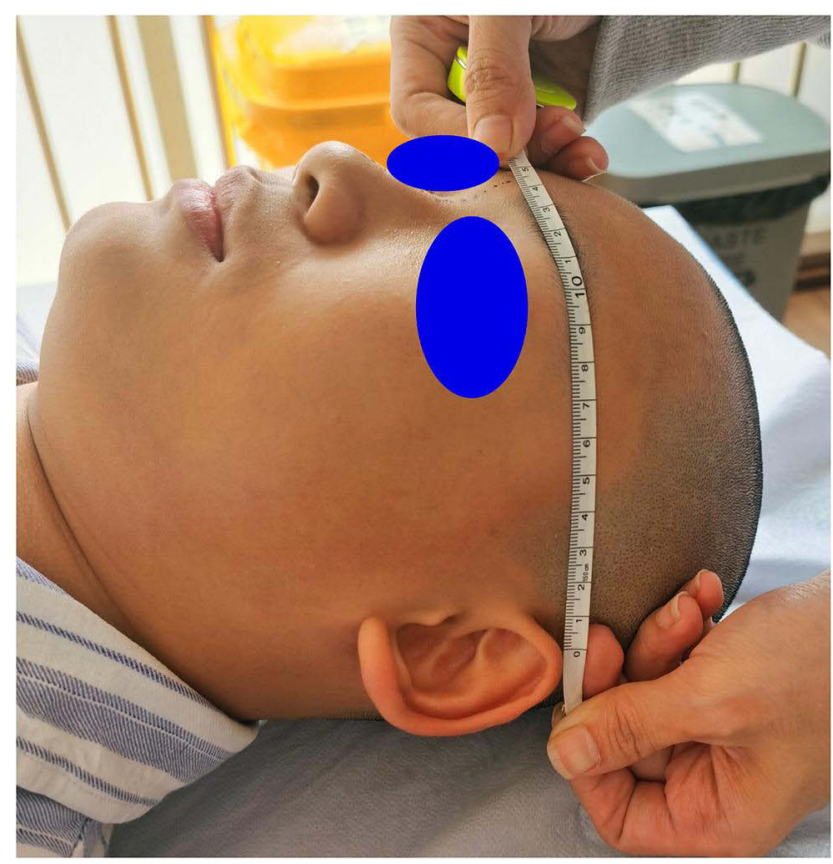

Figure 9 Patients were placed in supine position during measurement.

distance from the normal auricle to the central axis of the nose was $12.67 \pm 0.26 \mathrm{~cm}$. The average distance from the highest to the lowest point of the reconstructed auricle was 6.03 $\pm 0.32 \mathrm{~cm}$. The average distance from the highest to the lowest point of the normal auricle was $5.91 \pm 0.27 \mathrm{~cm}$. The results of the Shapiro-Wilk test indicated that the data distributions were non-normal $(P<0.05)$. According to the Wilcoxon signed-rank test, the distance from the highest point of the reconstructed auricle to the central axis of the nose was not significantly different from the distance from the highest point of the normal auricle to the central axis of the nose $(P>0.05)$; the distance from the lowest point of the reconstructed auricle to the central axis of the nose was not significantly different from the distance from the lowest point of the normal auricle to the central axis of the nose $(P>0.05)$; the distance from the highest to the lowest point of the reconstructed auricle was not significantly different from the distance from the highest to the lowest point of the normal auricle $(P>0.05)$. These results demonstrate that the reconstructed auricle was highly symmetrical to the contralateral auricle.

The data are summarized in Table 1. Postoperative images are shown in -Figure 15.

\section{Discussion}

After auricular reconstruction, the reconstructed and normal contralateral auricle should have the same size and position. ${ }^{10}$ Therefore, the accurate positioning of the reconstructed auricle preoperatively is essential. Auricular reconstruction is based on the experience of the surgeon and is subjective and uncontrollable. 3D

Table I Data Summary Table

\begin{tabular}{|c|c|c|}
\hline Variables & $\begin{array}{l}\text { Specific } \\
\text { Values }\end{array}$ & $\begin{array}{l}\text { Wilcoxon Signed Rank Test, } \\
\text { P-value }\end{array}$ \\
\hline Age, years & $10.77 \pm 2.64$ & \\
\hline \multicolumn{3}{|l|}{ Gender } \\
\hline Male & 29 (61.7\%) & \\
\hline Female & $18(38.3 \%)$ & \\
\hline Follow-up, months & $8.68 \pm 2.4$ & \\
\hline $\begin{array}{l}\text { The vertical distance from the highest point of the reconstructed auricle to the central axis } \\
\text { of the nose, } \mathrm{cm}\end{array}$ & $14.12 \pm 0.52$ & \multirow{2}{*}{$P=0.77(P>0.05)$} \\
\hline $\begin{array}{l}\text { The vertical distance from the highest point of the patient's normal auricle to the central axis } \\
\text { of the nose, } \mathrm{cm}\end{array}$ & $14.08 \pm 0.45$ & \\
\hline $\begin{array}{l}\text { The vertical distance from the lowest point of the reconstructed auricle to the central axis of } \\
\text { the nose, } \mathrm{cm}\end{array}$ & $|2.7| \pm 0.3 \mid$ & \multirow{2}{*}{$P=0.46(P>0.05)$} \\
\hline The vertical distance from the patient's normal auricle to the central axis of the nose, $\mathrm{cm}$ & $12.67 \pm 0.26$ & \\
\hline $\begin{array}{l}\text { The length of the line between the highest point and the lowest point of the reconstructed } \\
\text { auricle, } \mathrm{cm}\end{array}$ & $6.03 \pm 0.32$ & \multirow{2}{*}{$P=0 . I(P>0.05)$} \\
\hline $\begin{array}{l}\text { The length of the line between the highest point and the lowest point of the patient's normal } \\
\text { auricle, } \mathrm{cm}\end{array}$ & $5.91 \pm 0.27$ & \\
\hline
\end{tabular}




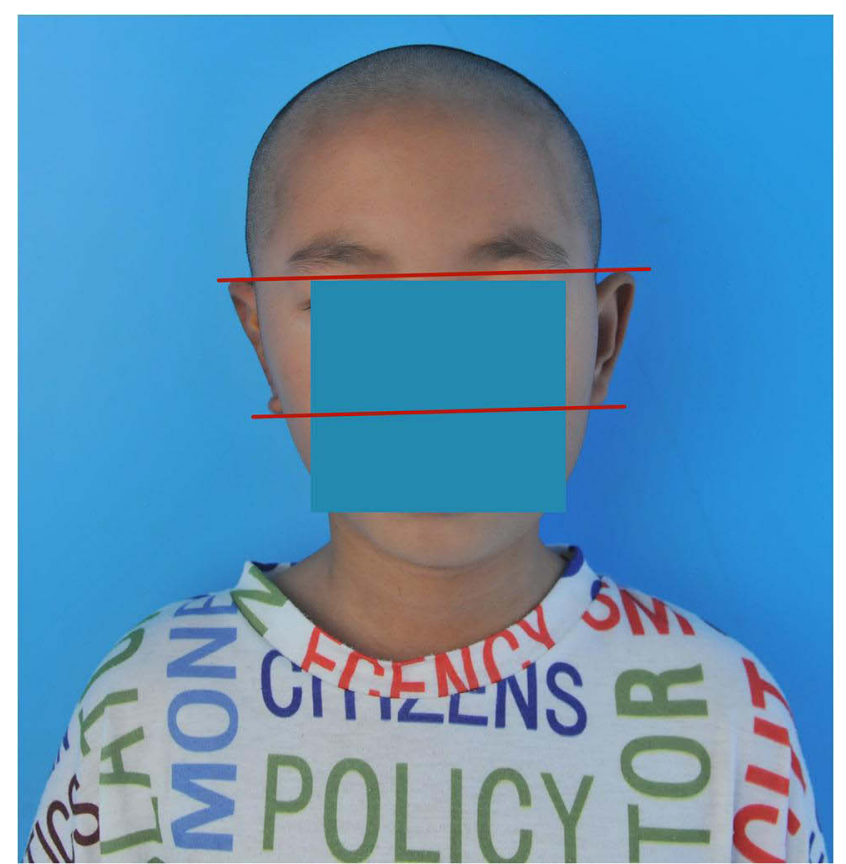

Figure 10 Frontal view of patient I.

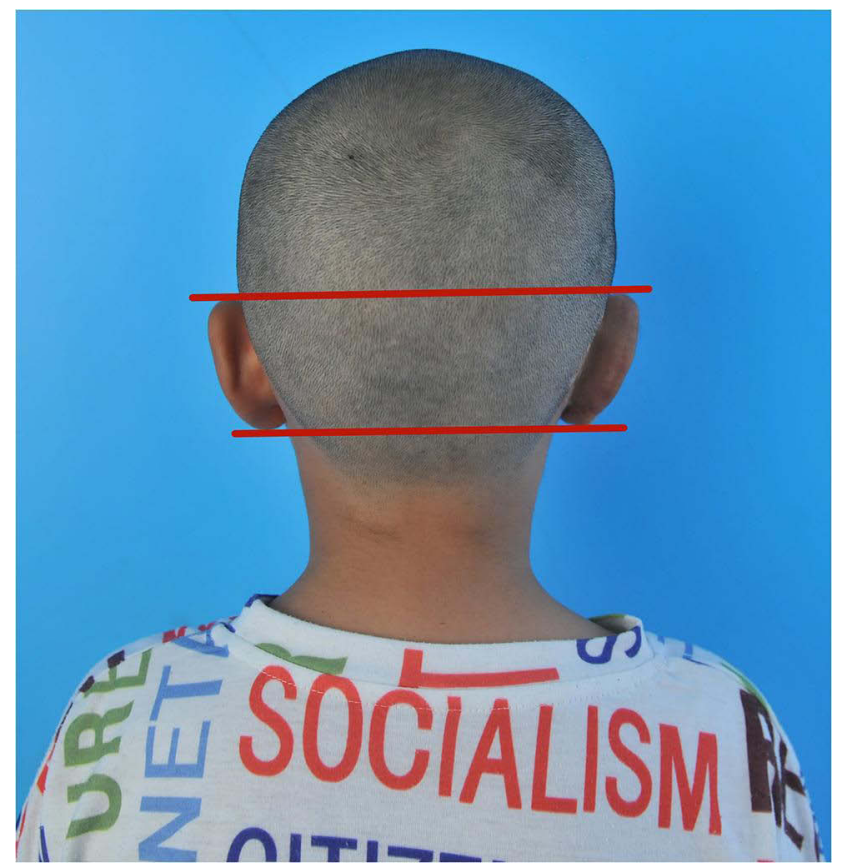

Figure I I Posterior view of patient I.

printers, CT equipment, and computer-assisted positioning method are complex and expensive, limiting their use in clinical practice. ${ }^{7-9}$ The ruler developed by our surgical team is simple, easy to use, accurate, and non-invasive and thus more applicable in plastic surgery.

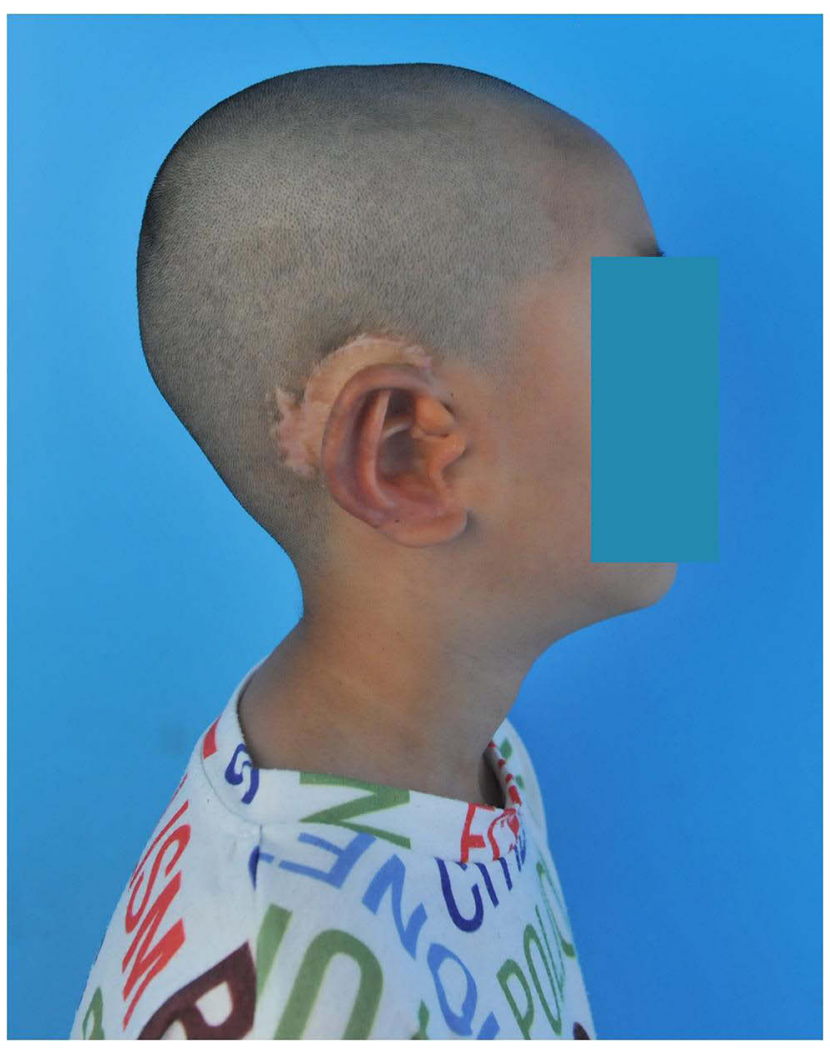

Figure 12 Lateral view of patient I.

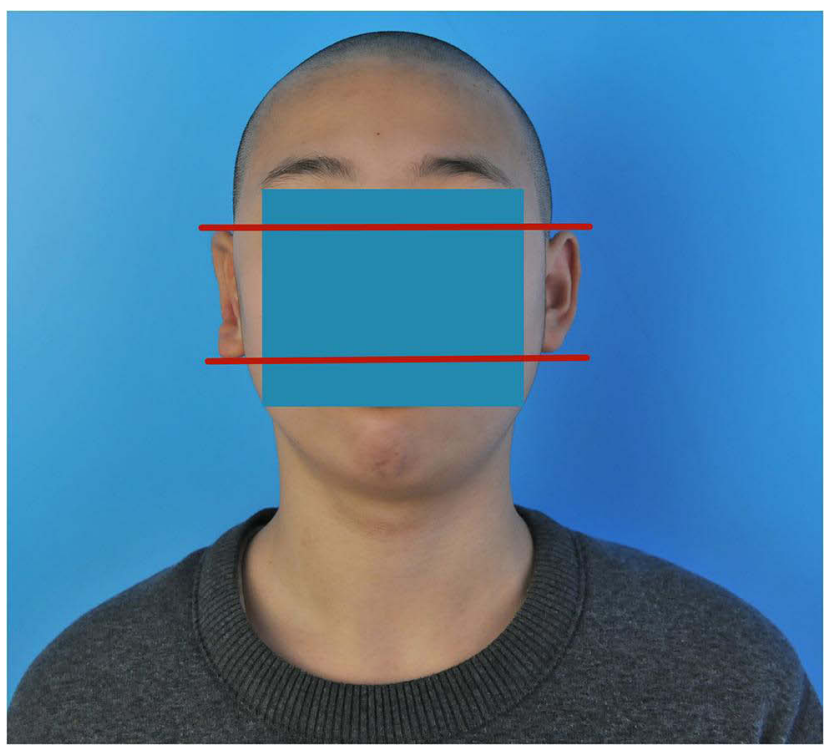

Figure 13 Frontal view of patient 2.

The positioning of auricular reconstruction was performed preoperatively in 47 patients using a ruler. The results showed that the reconstructed auricle was symmetrical to the normal contralateral auricle, confirming the accuracy of this instrument. 


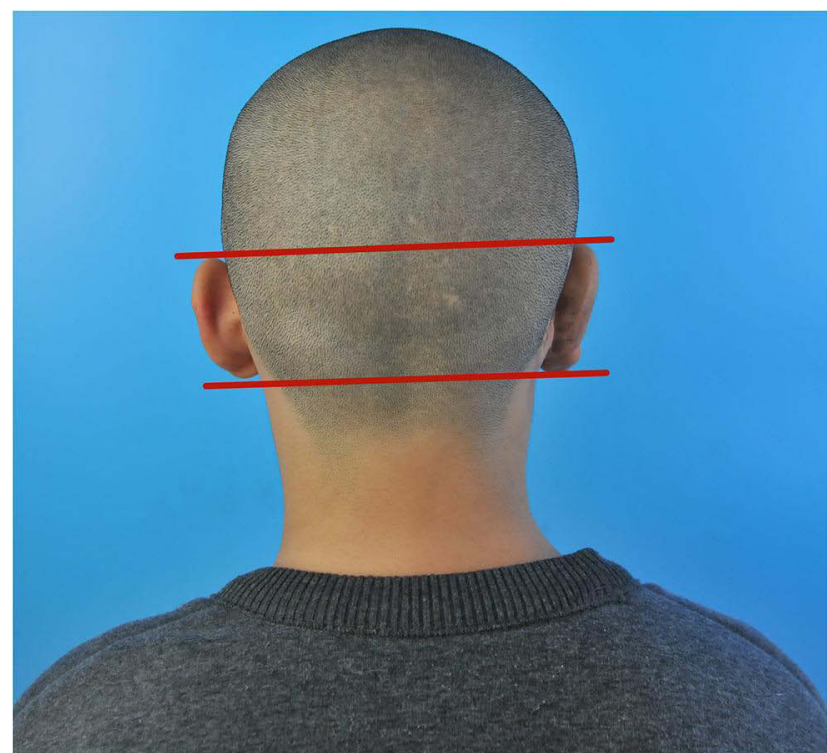

Figure I4 Posterior view of patient 2.

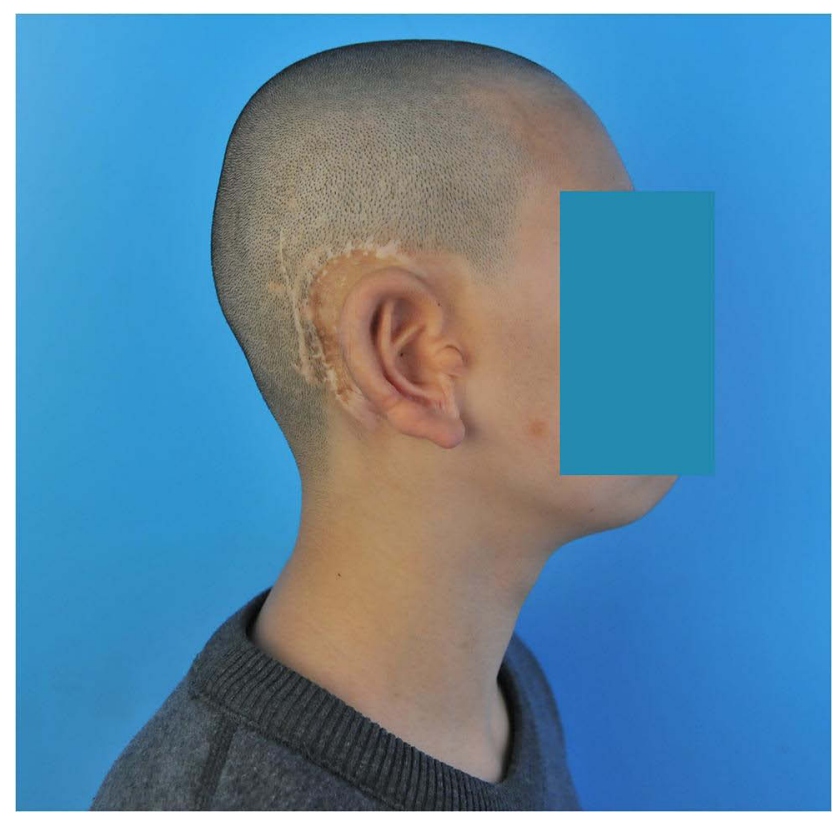

Figure I5 Lateral view of patient 2.

The clinical condition of the patients should be considered when positioning the reconstructed auricle using a ruler. Many patients with microtia have varying degrees of craniofacial asymmetry. ${ }^{11}$ In patients with severe craniofacial asymmetry, the plastic surgeon should move the reconstructed auricle slightly forward to adapt to the asymmetry. In addition, the positioning of the reconstructed auricle in patients with bilateral microtia should be carried out according to the ideal anatomical position of the auricle. The positioning ruler is suitable for all types of auricular reconstruction surgeries currently used in clinical practice. In two-stage or three-stage ear reconstruction surgeries, the ruler can be used to position the reconstructed auricle before each surgical stage and provide reference for surgeons' surgical operations.

This study has limitations. First, the small sample size may have affected the reliability of the results. Second, patients with severe craniofacial asymmetries or bilateral microtia were not included in the study; thus, the results do not apply to this population. In this respect, a follow-up study with a larger sample size is underway to assess the usefulness and accuracy of this ruler in cases involving severe craniofacial asymmetries or bilateral microtia. And in order to verify the advancement of positioning ruler, in subsequent studies, we will conduct comparative studies with other positioning methods. Third, researchers were not blinded to patient data, potentially increasing the risk of data collection bias.

In conclusion, the ruler evaluated in this study is simple, easy to use, accurate, and non-invasive, and thus highly useful in plastic surgery.

\section{Ethical Approval}

All procedures involving human participants conformed to the ethical guidelines of the institutional and national research committee and the 1964 Declaration of Helsinki. The study was approved by the Medical Ethics Committee of the Chinese Academy of Medical Sciences of the Plastic Surgery Hospital (No. 2021185).

\section{Acknowledgments}

The authors are grateful to all the patients for their active participation in this study.

\section{Funding}

This study is supported by the Institute Foundation of Plastic Surgery Hospital, Chinese Academy of Medical Sciences (3060120031).

\section{Disclosure}

The authors declare that there are no conflicts of interest associated with the research, authorship, and publication of this study.

\section{References}

1. Lu M, Lu X, Jiang H, Pan B. Review of preferential suspicious genes in microtia patients through various approaches. J Craniofac Surg. 2020;31(2):538-541. doi:10.1097/SCS.0000000000006244 
2. Guo F, Lin L, Yu X, et al. Classification of the concha-type microtia and their new suitable treatment strategies without autogenous costal cartilage grafting. Int J Pediatr Otorhinolaryngol. 2020;130:109801. doi:10.1016/j.ijporl.2019.109801

3. Luquetti DV, Leoncini E, Mastroiacovo P. Microtia-anotia: a global review of prevalence rates. Birth Defects Res a Clin Mol Teratol. 2011;91(9):813-822. doi:10.1002/bdra.20836

4. Yang M, Jiang H, Zhang Y, Pan B. A new method using tissue expander makes auricular reconstruction easier. Clin Otolaryngol. 2019;44(3):437-442. doi:10.1111/coa.13304

5. Nagata S. Modification of the stages in total reconstruction of the auricle: part I. Grafting the three-dimensional costal cartilage framework for lobule-type microtia. Plast Reconstr Surg. 1994;93 (2):221-230. doi:10.1097/00006534-199402000-00001

6. Yamada A. Autologous rib microtia construction: Nagata technique. Facial Plast Surg Clin North Am. 2018;26(1):41-55. doi:10.1016/j. fsc.2017.09.006
7. Chen K, Fu Y, Yang L, Dai P, Zhang T, New Three-Dimensional A. Template for the fabrication and localization of an autogenous cartilage framework during microtia reconstruction. ORL $J$ Otorhinolaryngol Relat Spec. 2015;77(3):150-154. doi:10.1159/000381919

8. Umekawa K. Deciding on the position for auricular reconstruction. Adv Otorhinolaryngol. 2014;75:39-43. doi:10.1159/000350601

9. Walsh WE, Reisberg DJ, Danahey DG. A new device for creating and positioning an autogenous cartilage framework during microtia reconstruction. Laryngoscope. 2005;115(11):2068-2071. doi:10.1097/ 01.mlg.0000178326.73300.42

10. Nuri T, Mitsuno D, Otsuki Y, Ueda K. Augmented reality technology for the positioning of the auricle in the treatment of microtia. Plast Reconstr Surg Glob Open. 2020;8(2):e2626. doi:10.1097/ GOX.0000000000002626

11. Wang Y, He L, Jiang H, et al. Digital model simulation technology for ear reconstruction of microtia with craniofacial asymmetry. J Plast Reconstr Aesthet Surg. 2019;72(4):685-710. doi:10.1016/j.bjps.2019.01.028

\section{Publish your work in this journal}

Therapeutics and Clinical Risk Management is an international, peerreviewed journal of clinical therapeutics and risk management, focusing on concise rapid reporting of clinical studies in all therapeutic areas, outcomes, safety, and programs for the effective, safe, and sustained use of medicines. This journal is indexed on PubMed Central, CAS,
EMBase, Scopus and the Elsevier Bibliographic databases. The manuscript management system is completely online and includes a very quick and fair peer-review system, which is all easy to use. Visit http://www.dovepress.com/testimonials.php to read real quotes from published authors. 